\title{
As práticas de sistemas CAD e sua contribuição: um survey na indústria metal-mecânica mineira
}

\author{
André César de Figueiredo ${ }^{\mathrm{a}, *}$, Eduardo Romeiro Filho ${ }^{\mathrm{b}}$ \\ a,*acf@ufmg.br, UFMG, Brasil \\ bromeiro@ufmg.br, UFMG, Brasil
}

\begin{abstract}
Resumo
Este artigo apresenta os resultados de uma enquete descritiva (survey) para avaliação da utilização de sistemas CAD (Computer Aided Design, ou Projeto Auxiliado por Computador) em setores de projeto de produto de organizações do setor metal-mecânico no Estado de Minas Gerais. Como resultado do survey, verificou-se que há diferenças entre as aplicações CAD descritas pela literatura e as práticas levantadas nas organizações pesquisadas, consistindo um conjunto de características específicas à região e ao setor estudado. Além disso, problemas apontados pela literatura (como questões posturais e alto custo de equipamentos e treinamento) foram observados na pesquisa, o que pode indicar que a situação atual das aplicações CAD nas empresas pode ser melhorada a partir de uma abordagem adequada, fundamentada a partir do levantamento da situação e das limitações existentes.
\end{abstract}

Palavras-chave

CAD. Projeto do produto. Survey. Setor metal-mecânico.

\section{Introdução}

Diferentes autores, como Griffin e Page (1996), Griffin (1997) e Fleury (1999) definem que os fatores de competição industrial são baseados em aspectos como custo, qualidade, tempo, flexibilidade e inovação. Como demonstram os resultados dos indicadores pesquisados pela CNI e Sebrae (CONFEDERAÇÃO..., 2005), a indústria brasileira tem se dedicado a implementar modificações e melhorias nessas dimensões competitivas. Esses indicadores constataram um cenário positivo no que diz respeito aos investimentos realizados na atividade de $\mathrm{P} \& \mathrm{D}$ e à disposição por parte dos estabelecimentos industriais de adquirir máquinas e equipamentos. Não por acaso, diversas dessas áreas encontram endosso em autores consagrados como Clark e Fujimoto (1991), Clark e Wheelwright (1993), Urban e Hauser (1993), Roozenburg e Eekels (1995) e Ulrich e Eppinger (2000).

Os sistemas CAD reconhecidamente contribuem para a inovação nas empresas, em especial no desenvolvimento de novos produtos industriais em diferentes áreas como descrito por Qiu et al. (2007),
Stamati e Fudos (2005), Nagalingam e Lin (2008) e Dankwort et al. (2004), por exemplo. A Jon Peddie Research (2010) estima que o mercado mundial de sistemas CAD tenha atingido US\$ 5 bilhões em 2009, o que representa uma queda de $23 \% \mathrm{em}$ relação a 2008 (período anterior à crise financeira), quando foram atingidos US\$ 6,7 bilhões. Desse total, aproximadamente metade refere-se à indústria metal-mecânica, o que demonstra a importância de pesquisas voltadas para o setor. Faz-se dessa forma oportuna uma investigação sobre as aplicações nas indústrias brasileiras, bem como das vantagens e desvantagens observadas por estas. Assim será possível compreender as práticas adotadas por usuários de sistemas CAD, e também suas dificuldades e necessidades, o que é essencial para o desenvolvimento de soluções de engenharia ou gestão que atendam às características peculiares das empresas brasileiras.

Buscando contribuir para essa compreensão, este artigo apresenta uma pesquisa das práticas em sistemas CAD em empresas metal-mecânicas, 
realizada no Estado de Minas Gerais, maior em extensão da região Sudeste e terceiro PIB nacional. 0 estado apresenta um potencial econômico expressivo, o que pode ser demonstrado pelo fato de ocupar uma posição de liderança em diversos produtos importantes para a economia nacional e internacional, tanto em atividades tradicionais como em setores de ponta. Dentro desse contexto, a indústria de transformação responde pelo maior número de empresas e empregados dentro do setor industrial no estado, ganhando assim grande notoriedade e visibilidade.

A partir desse cenário, justifica-se o foco deste trabalho na indústria mecânica ou metal-mecânica do Estado de Minas Gerais que, segundo dados da FIEMG (FEDERAÇÃO..., 2006a), responde por 1.657 estabelecimentos industriais, empregando mais de 23 mil pessoas. Soma-se a isso o crescimento no faturamento desse setor nos primeiros meses do ano de 2006 e a elevação nas vendas desse setor para o mercado interno, como aponta a própria FIEMG (FEDERAÇÃO..., 2006b). Dessa maneira, trata-se de um setor com grande representatividade e significativa participação na economia mineira. Por fim, demonstra-se adequado para a pesquisa, pois a indústria metal-mecânica é tradicionalmente grande usuária de sistemas CAD em todo o mundo.

\section{Sistemas informatizados de auxílio ao projeto - CAD}

No projeto, o emprego do processamento de dados e da tecnologia da informação serve tanto para a melhoria do produto como para a redução do custo do projeto e de produção (PAHL; BEITZ, 1996; PAHL et al., 2005). Com isso, esses autores definem CAD como a técnica do trabalho de projeto interligada ao uso do computador mediante aproveitamento de periféricos e programas apropriados. Rehg e Kraebber (2005) afirmam que CAD é a aplicação de computadores e softwares gráficos para auxiliar ou melhorar o projeto de produto desde a conceituação até a documentação, suportando todos os níveis no processo de projeto de produto. Esses autores ainda complementam colocando que o CAD é a principal tecnologia para um processo integrado de desenvolvimento de produto em uma manufatura integrada por computador (CIM). Os autores colocam seis áreas em que os sistemas CAD podem ser utilizados: a) projeto de produto; b) análise de produto; c) avaliação de produto; d) desenvolvimento da documentação; e) engenharia de produção; e f) engenharia de manufatura.
Há que se levar em consideração a colocação de Romeiro Filho (1997), onde a utilização de sistemas CAD pode auxiliar em muito a difusão e intercâmbio de informações em tempo real, a grandes distâncias, articulando diferentes equipes de projeto, mesmo em empresas distintas. Nesse caso, a empresa que desenvolve 0 projeto poderá consultar seus fornecedores a respeito das características mecânicas de determinada peça, bem como sugerir alterações pertinentes, por exemplo. Esses aspectos nos levam a uma abordagem contemporânea dos sistemas $C A D$, que atuam menos como ferramentas de projeto e mais como elementos de integração das diferentes áreas de uma ou de várias empresas. Com efeito, a maioria dos artigos relacionados à engenharia simultânea faz menção à aplicação de sistemas CAD.

A partir da revisão de literatura (QIU et al., 2007, STAMATI; FUDOS, 2005, NAGALINGAM; LIN, 2008, DANKWORT et al., 2004, por exemplo), percebe-se que as soluções técnicas existentes no mercado atual podem atender (ao menos teoricamente) de forma bastante extensa às várias necessidades de projeto. Não somente em se tratando de ferramentas de apoio direto, como sistemas CAD, mas principalmente com o desenvolvimento de soluções para auxílio a atividades complementares, como sistemas de apoio ao trabalho em grupo, ou workgroup; sistemas de gerenciamento de documentos (como os sistemas EDI e EDMS), para transmissão de dados on-line, além dos vários sistemas de apoio computadorizado às atividades da empresa, como CAE, CAM, CAPP etc., que levam a perspectiva de um processo integrado de projeto, documentação e gerenciamento de informações relacionadas a produtos e processos por meio de sistemas informatizados, dentro de um conceito mais amplo e sistêmico, de PLM (Product Lifecycle Managment, ou gestão do ciclo de vida do produto).

0 PLM possui três conceitos fundamentais (CIMDATA, 2009): a) manter a integridade do produto, a definição e as informações relacionadas ao longo da vida do produto; b) manter as informações do produto universais e seguras; e c) garantir a gestão e manutenção dos processos empresariais utilizados para criar, gerenciar, divulgar, partilhar e utilizar a informação. As necessidades de gerenciamento de dados do produto dependem muito das características da empresa. Muitos sistemas têm evoluído para satisfazer as necessidades não só de grandes empresas, mas também de pequenas e médias empresas de todos os tipos. Essa evolução (AMANN, 2004) é um sinal da aceitação geral do mercado da tecnologia de gestão de dados e do PLM, o qual além dos tradicionais CAD/CAE/ 
CAM possui como elementos: tecnologias e normas (visualização, colaboração, Enterprise Application Integration etc.), informações e ferramentas de análise, funções essenciais (documentos e gestão de conteúdos, gerenciamento workflow, classificação e programa de gestão), além de integração com outros processos de negócios como o Customer Relationship Management e o Supply Chain Management.

McEleney (2006) define o PLM como uma estratégia de reengenharia de processos destinada a aproveitar os dados de design (gerados pelo (AD) e outras tecnologias, como os sistemas de planejamento de recursos empresariais (ERP Enterprise Resource Planning), para gerenciar ciclos de vida de produtos, refazer processos e aprimorar a produção, com as melhorias de produtividade vindas de toda a empresa, ao invés de um único departamento ou de um processo específico. O PLM é visto como uma ferramenta que gerencia dados de design e engenharia de produtos, para automatizar o desenvolvimento dos produtos, aumentando a eficiência nos processos existentes. Dentro desse conceito, o PDM (Product Data Management, ou gestão de informações do produto) pode ser entendido como parte integrante do PLM, sendo associado a aplicações CAD mais avançadas.

\section{Método de pesquisa}

Forza (2002) afirma que, em geral, uma pesquisa por enquete (survey) envolve a coleção de informações vindas dos indivíduos (seja através de questionários por correio, chamadas telefônicas, entrevistas pessoais etc.) sobre eles mesmos ou às unidades sociais a que pertencem. Assim, podem-se coletar informações sobre uma grande população com um conhecido nível de precisão. Forza (2002), Pinsonneault e Kraemer (1993) atribuem três características básicas à pesquisa por enquete. Primeiramente, ela se propõe a fornecer descrições quantitativas de determinados aspectos da população estudada, que pode consistir em estabelecer relações entre variáveis ou em realizar projeções. Em segundo lugar, a coleta de dados é realizada, via de regra, a partir de questionários estruturados e predefinidos. As respostas a essas questões constituem os dados que são analisados. E por último, as informações são em geral coletadas junto a uma fração, ou amostra, da população-alvo.

Pinsonneault e Kraemer (1993) ressaltam que: a) a pesquisa por enquete é particularmente apropriada quando a questão de pesquisa diz respeito ao quê, ao por que se dá e ao como se dá um fenômeno dado; b) o controle das variáveis dependentes e independentes geralmente é inútil e inviável; c) o fenômeno pesquisado deve ocorrer no momento presente ou num passado recente e é estudado em seu ambiente natural. Essas colocações feitas por esses autores encontram-se em consonância com as questões e variáveis levantadas na revisão de literatura em relação ao fenômeno "sistemas CAD" (KRISHNAN; ULRICH, 2001) e com os objetivos fixados para tratar desse fenômeno ao longo deste artigo.

Portanto, para esta pesquisa, adotou-se um caráter amostral, já que, em análise preliminar, verificou-se que o número de elementos da população em estudo (organizações do setor metalmecânico do Estado de Minas Gerais) é elevado. Optou-se pelo uso da amostragem aleatória simples para seleção dos elementos da população estudada. Para o mapeamento e a localização dos elementos de população do survey, utilizou-se o Cadastro Industrial de Minas Gerais, desenvolvido pelo CIEMG (Centro Empresarial do Estado de Minas Gerais) e pelo sistema FIEMG (Federação das Indústrias do Estado de Minas Gerais). Nesse cadastro (em sua versão 2005) há um total de 16.056 empresas catalogadas, divididas por áreas de atuação, sendo 1.780 pertencentes ao setor metalmecânico. Como a pesquisa se dedica ao estudo de sistemas informatizados, o que pressupõe a utilização de computadores e meios informatizados de comunicação, retirou-se da lista as empresa que não possuíam correio eletrônico. Assim o número de empresas reduziu-se para 1.134 (população do survey). Seguindo o que colocam Sheaffer et al. (1990) e Barbetta (2002) para cálculo do tamanho da amostra dentro do rigor estatístico, tendo que o tamanho da população é de 1.134 elementos e admitindo um erro amostral considerado tolerável de $15 \%$, a amostra definida é de aproximadamente 45 empresas.

0 processo de amostragem utilizado permite obter um tamanho mínimo de amostra com base numa medida de precisão preestabelecida. De acordo com Sheaffer, Mendenhall e Otto (1990) e Barbetta (2002), para a obtenção do tamanho mínimo da amostra definem-se uma medida de erro d (diferença observada entre a proporção verdadeira e a estimada) e o nível de confiança desejado que satisfaçam à Equação 1, onde $P$ é a proporção observada na população; $\hat{P}$ a proporção estimada; $d$ a margem de erro; e $(1-\alpha)$ é o nível de confiança.

$\operatorname{Prob}\{|\hat{P}-P| \leq d\}=1-\alpha$

Assim, de acordo com os autores, o tamanho da amostra (n) deverá é calculado pela Equação 2, 
onde N é o tamanho da população; $z_{\alpha} 0$ valor da tabela normal associada ao nível de confiança desejado; P é a proporção de respostas positivas; e $(1-\mathrm{P})$ a proporção de respostas negativas.

$n=\frac{N \cdot z_{\alpha}^{2} \cdot P(1-P)}{N \cdot d^{2}+z_{\alpha}^{2} \cdot P(1-P)}$

Dado que a proporção observada na população não é conhecida, adotou-se que as proporções de respostas positivas e negativas sejam as mesmas, ou seja, $\mathrm{P}=(1-\mathrm{P})=50 \%$. Esse procedimento faz com que o tamanho da amostra seja maximizado para o nível de confiança e margem de erro preestabelecidos.

Dessa maneira, para se determinar o tamanho mínimo da amostra foram feitas algumas considerações a respeito do nível de confiança e da margem de erro que seriam adotados pela pesquisa.

Optou-se pela aplicação de um questionário, elaborado a partir da revisão da literatura pertinente ao tema, com o objetivo de construir as bases conceituais e o quadro teórico de referência. 0 instrumento de coleta de dados foi estruturado com base em tópicos principais (divididos em subtópicos) como: a) identificação da empresa; b) responsável pelas informações; c) sistema CAD na empresa; d) aprendizado em CAD e sua utilização; e e) organização do trabalho. Seguiu-se neste trabalho o que foi feito por Soliman e Clegg (2001) para a validação do conteúdo do questionário: a) inspeção da literatura para todos os possíveis itens a serem incluídos na pesquisa; b) críticas à descrição e clareza do questionário por um grupo de acadêmicos e professores de Engenharia de Produção; c) teste do questionário em um grupo foco com profissionais e acadêmicos; e d) modificação do questionário baseada nesses três itens antes da realização da pesquisa.

Elaboraram-se as correspondências de encaminhamento do instrumento à empresa, dirigida ao profissional responsável pelo sistema CAD, discriminando os objetivos da pesquisa e sua relevância, a instituição e os organismos realizadores, a importância da colaboração do respondente, os procedimentos relativos ao preenchimento, o registro do compromisso de resguardar a confidencialidade dos dados e dos resultados isolados e do compromisso de - uma vez concluídas a pesquisa - remeter cópia do sumário executivo dos resultados conquistados para o respondente. Utilizaram-se procedimentos mistos para a realização da pesquisa como forma de ampliar e enriquecer a visão acerca do fenômeno estudado. Assim, para a aplicação do questionário, foram utilizados o contato telefônico, o envio de correspondência eletrônica, a visita seguida da aplicação in-loco e a elaboração de um site. Este foi desenvolvido como meio de propiciar à população do survey distribuída geograficamente pelo Estado de Minas Gerais a possibilidade de utilizar a internet para responder o questionário de pesquisa. Desenvolvido em linguagem HTML, também estava associado a um banco de dados para ser utilizado como centralizador dos dados coletados pelos outros procedimentos de pesquisa, sendo que isso se destinava a facilitar a seleção e o tratamento dos dados.

\section{Resultados, análise e discussão}

A pesquisa demonstrou que a maior parte $(87 \%)$ das empresas pesquisadas se caracteriza como estabelecimento industrial. 0 percentual restante é composto por organizações que se ligam às indústrias por meio de prestação de serviço. No tocante ao porte das empresas, o survey parece ter sido composto por uma amostra bastante equilibrada no que se refere à participação de grandes e médias (40\% de cada grupo de empresas). E a amostra ainda contou com 13\% de micro empresas e 7\% de pequenas empresas. Dessa maneira, pode-se dizer que o surveyconseguiu abranger um espectro amplo de organizações do ponto de vista do porte do estabelecimento, tentando retratar o que acontece realmente no setor pesquisado. A maior parte das empresas pesquisadas (cerca de 90\%) destina seus produtos para o mercado nacional e metade delas (50\%) exporta seus produtos. Isso parece indicar que as empresas do setor metal-mecânico estão superando os desafios colocados por Rembold, Nnaji e Storr (1993) e Fleury (1999), destinando seus produtos para mercados onde encontram mais concorrentes e níveis superiores de exigência.

Verificou-se que a maior parte dos entrevistados $(60 \%)$ tem mais de 36 anos e que cerca de 70\% tem formação superior (33\% desses têm pós-graduação), sendo que 54\% dos pesquisados são engenheiros. Daí, pode-se inferir a existência de uma tendência em colocar no quadro de recursos humanos, na área de projeto das organizações do setor metalmecânico, trabalhadores qualificados com formação superior e experiência em projeto e CAD. Essa dedução pode também ser validada ao considerar que cerca de 51\% dos entrevistados tem mais de 10 anos de formado; 74\% trabalham com projeto há seis ou mais anos e $90 \%$ das pessoas pesquisadas têm três ou mais anos como usuário de sistemas CAD. E ainda, tal conclusão bate perfeitamente com o que colocam Neves, Caulliraux e Souza 
(1998) no que se refere a trabalhadores mais bem qualificados atuando com sistemas informatizados. Todavia, tempo na empresa foi uma variável sobre os pesquisados que acabou assumindo importância frente às demais, pois indica o tempo que essas pessoas estão empregadas na empresa sobre a qual responderam o questionário desta pesquisa survey, e, assim, pode-se verificar se esse tempo é minimamente adequado ou suficiente para que tais pessoas tenham conhecimento a respeito da empresa, e sobretudo da área de projeto em que trabalham para que os dados obtidos retratem mais fielmente a realidade. Dessa maneira, verificou-se que 71\% dos entrevistados têm três ou mais anos de tempo na empresa; o que pareceu bastante adequado para este trabalho. Um resultado significativo sobre os pesquisados a respeito de sistemas $C A D$ foi que a grande maioria (97\%) dos respondentes considera que a adoção dos sistemas CAD por empresas para uso em projeto é fundamental ou muito importante. Essa postura encontra endosso em vários autores aqui referenciados que tratam de sistemas CAD, como Scheer (1993), Rembold, Nnaji e Storr (1993), Anumba (1996), Malhotra, Heine e Grover (2001), Pahl et al. (2005).

\subsection{CAD e seu papel nas empresas}

No que se refere aos esforços empreendidos em projeto nas empresas, a pesquisa constatou que a maior parte das empresas pesquisadas dedica significativa colaboração da alta administração com as atividades desenvolvidas na área de projeto, sendo que 63\% das empresas têm um alto e 30\% delas têm médio comprometimento por parte da alta administração nos esforços de projeto. 0 que parece ser claro reflexo da conjuntura brasileira, em que empresários se dão conta do que colocou Davies (1997), de que o projeto se torna o primeiro passo na satisfação das exigências do cliente. A necessidade desse comprometimento da alta administração com as atividades de projeto como meio para a competitividade e para o desenvolvimento bem-sucedido de produtos é apontada por vários autores, como Clark e Fujimoto (1991), Clark e Wheelwright (1993), Urban e Hauser (1993), Roozenburg e Eekels (1995), Ulrich e Eppinger (2000) e Pahl et al. (2005). Entretanto, esse comprometimento da alta administração não beneficiou a área de projeto dentro da empresa no tocante à hierarquia e ao organograma: apurou-se que em apenas 10\% o projeto e os sistemas CAD se elevam ao nível de diretoria. Dessa maneira, a área de projeto nas empresas é organizada como uma chefia ou uma gerência; em 37\% delas, o projeto e os sistemas $C A D$ se vinculam a uma chefia e, em 53\% das empresas, o projeto e os sistemas CAD se estruturam em torno de uma gerência dentro da empresa. Da mesma forma que o comprometimento da alta administração não favoreceu a área de projeto no tocante à hierarquia, parece ter acontecido o mesmo no que se refere à quantidade de empregados envolvidos em projeto, já que não houve uma definição clara do número de funcionários que participaram. No tocante à adoção dos sistemas CAD e tempo de utilização desses sistemas, averiguou-se que a maioria das empresas (73\%) utiliza sistemas CAD há seis ou mais anos.

\subsection{Sistemas informatizados adotados em projeto}

Neste trabalho, verificaram-se quais são os sistemas ou softwares CAD adotados pelas empresas para uso em suas atividades de projeto a partir de uma lista desses sistemas ou softwares CAD comumente comercializados no mercado de tecnologia. Constatou-se ainda uma predominância de sistemas CAD low-end (de baixo custo, ou da "base" do mercado); sendo que 66,70\% das empresas adotam como software o AutoCAD ${ }^{\mathrm{TM}}$. Entretanto, parece haver uma segmentação de mercado e a entrada de outros sistemas low-end, já que 43,30\% das empresas adotam outros sistemas CAD, sendo os mais citados o SolidEdge ${ }^{\mathrm{TM}}$ e o SolidWorks ${ }^{\mathrm{TM}}$ (o fato de a soma exceder 100\% é porque algumas empresas utilizam mais de um sistema (AD). Tomando as colocações de Rosenthal (1984) e Taffs (1997), as empresas do setor metal-mecânico parecem ter optado por sistemas CAD em que se pode verificar a confiabilidade já comprovada do fornecedor do sistema na área, a disponibilidade de serviço de manutenção e o baixo custo do software; e levando em consideração que o estudo empreendido por Figueiredo, Lacerda e Romeiro (2003) indicou que os sistemas citados atendem a esses três requisitos dentro do Estado de Minas Gerais. Contudo, há indícios de que a utilização de sistemas high-end (do "topo" do mercado, representando altos custos de aquisição, treinamento e manutenção) vem sofrendo incrementos; pois, 13,30\% das empresas utilizam o sistema CATIA $^{\mathrm{TM}}, 13,30 \%$ usam sistema UGS $^{\mathrm{TM}}, \quad 6,70 \%$ adotam o Microstation $^{\mathrm{TM}}, \quad 3,30 \%$ utilizam o sistema Pro-Engineer ${ }^{\mathrm{TM}}$.

\subsection{Aplicações dos sistemas $C A D$}

As principais aplicações desempenhadas pelos sistemas CAD dentro das empresas constituem um dos aspectos mais importantes a serem tratados quando se busca compreender o universo dos 
sistemas CAD. Como o foco deste trabalho são as empresas do setor mecânico/metal-mecânico, verificou-se, obviamente, que, em 96,70\% delas, os sistemas CAD na área de projeto são utilizados para desenvolver desenho mecânico. E outros tipos de desenhos também são desenvolvidos em tais sistemas nas empresas; em 43,30\%, são feitos desenhos eletro-eletrônicos; em 33,30\%, fazem desenhos de tubulações; em 30\%, desenhos civis ou de arquitetura são desenvolvidos; e em 26,70\%, desenhos de hidráulica.

Com esses dados, pode-se dizer que as empresas dominam uma aplicação básica dos sistemas CAD: a construção de elementos tridimensionais de produtos por meios computacionais (por meio de uma modelagem geométrica ou "geometria computacional"), como apontam diversos autores (CAULLIRAUX; COSTA, 1995, ANUMBA, 1996, LUONG, 1998, MALHOTRA; HEINE; GROVER, 2001, DANKWORT et al., 2004). Dessa maneira, as empresas fazem uso da modelagem geométrica para a realização de projetos mecânicos e para a sua complementação com desenhos auxiliares de outras áreas, como eletro-eletrônicos, civis, hidráulicos etc. Contudo, esses mesmos autores indicam uma série de aplicações mais complexas e amplas para os sistemas CAD. E isso é o que parece fazer uma parcela relevante das empresas, já que 33,30\% utilizam CAD para desenvolver simulações e maquetes eletrônicas; 30\% usam CAD com aplicações CAM (manufatura auxiliada por computador), integrando o CAD com a produção e ligando-o a máquinas CNC (controle por comando numérico); e, também, 30\% delas desenvolvem aplicações CAE (engenharia auxiliada por computador), realizando cálculos de engenharia através de sistemas CAD. Soma-se a isso que em $43,30 \%$ das empresas há a troca de dados com outras estações; e que em 33,30\% há troca de dados a distância via rede de telecomunicações. Entretanto, 26,70\% das empresas usam os sistemas CAD para a elaboração de textos, geração de gráficos e/ou planilhas; e ainda 3,30\% fazem outras aplicações dos sistemas CAD. Isso parece demonstrar uma subutilização dos sistemas CAD por parte de algumas empresas, o que remete aos baixos níveis de integração e utilização alcançados com sistemas CAD no Brasil, como já apontavam Caulliraux e Costa (1995) e Romeiro Filho (1997) em meados dos anos 1990.

\subsection{Vantagens na utilização dos sistemas $C A D$}

A grande maioria dos pesquisados considerou que as vantagens da adoção de sistemas CAD na área de projeto da empresa são muito expressivas, o que reforça o papel fundamental assumido pela utilização de sistemas CAD para projeto de produtos dentro das organizações pesquisadas. A Tabela 1 apresenta os resultados da enquete.

Essas vantagens se relacionam a aspectos de tempo (obviamente, com elas diminui o tempo gasto para a realização de projetos), de qualidade (ao integrar as várias fases do projeto, ao reduzir o trabalho de desenho, ao avaliar desenhos alternativos, melhora-se a qualidade do projeto e busca-se garantir a qualidade dos produtos), de custo (consegue-se reduzir os custos de projeto e produção ao utilizar partes comuns em múltiplos produtos, ao garantir a precisão e qualidade do projeto), de flexibilidade (utilizando menos tempo na elaboração, pode-se usar o tempo restante na busca por soluções alternativas para o projeto ou para revisões e alterações), de comunicação e integração (ao integrar as várias fases do projeto por meio do sistema CAD, estabelece-se novas formas de comunicação que buscam ser mais eficazes e ágeis na área de projeto, como no caso do PLM) e inovação (aumentando o tempo empenhado na busca por soluções alternativas, estabelecendo novas formas de comunicação por meio do sistema CAD e aumentando a qualidade no projeto; graças a esse incremento na troca de informações, favorece-se a inovação). Essas vantagens também foram apontadas por vários autores, entre eles: Anumba (1996), Caulliraux e Costa (1995), Malhotra, Heine e Grover (2001), Pahl et al. (2005).

Esses mesmos autores indicam que, com a utilização de sistemas CAD em projeto nas empresas, deve haver melhoria das comunicações entre o pessoal de projeto e também no fluxo de informações através da empresa, já que o CAD propicia novas linhas de comunicação e busca integrar as fases e os esforços de projeto; associando-se a isso a possibilidade de centralizar as informações de projeto com sistemas CAD, como forma de simplificar a execução de atividades de projeto. Tais vantagens foram examinadas por meio do survey. No que se refere à melhoria das comunicações entre o pessoal de projeto, constatou-se que, em 67\% das empresas, houve esse aprimoramento; porém, em $20 \%$ delas não ocorreu melhoria das comunicações entre o pessoal de projeto; e ainda em 13\% não se aplica tal vantagem relacionada aos sistemas CAD.

Já a melhoria no fluxo de informações através da empresa foi observada em 70\% das empresas; entretanto em 17\% delas não houve benefício no fluxo de informações; e ainda em 13\% não se aplica tal vantagem em relação aos sistemas CAD. Ou seja, apesar de a maioria das empresas verificarem 
Tabela 1. Vantagens na utilização do CAD nas empresas pesquisadas.

\begin{tabular}{|c|c|c|c|c|c|c|c|c|}
\hline \multirow[t]{2}{*}{ Principais vantagens dos sistemas CAD na empresa } & \multicolumn{2}{|c|}{ Sim } & \multicolumn{2}{|c|}{ Não } & \multicolumn{2}{|c|}{$\begin{array}{l}\text { Não se } \\
\text { aplica }\end{array}$} & \multicolumn{2}{|c|}{ Não sabe } \\
\hline & Qtd & $\%$ & Qtd & $\%$ & Qtd & $\%$ & Qtd & $\%$ \\
\hline Maior velocidade na elaboração de projetos & 28 & 96,6 & 0 & 0,0 & 1 & 3,4 & 0 & 0,0 \\
\hline Maior integração entre as várias fases do projeto & 24 & 82,8 & 0 & 0,0 & 5 & 17,2 & 0 & 0,0 \\
\hline Padronização de métodos e procedimentos & 24 & 82,8 & 2 & 6,9 & 2 & 6,9 & 1 & 3,4 \\
\hline Redução no trabalho de desenho & 27 & 93,1 & 2 & 6,9 & 0 & 0,0 & 0 & 0,0 \\
\hline Redução do tempo e maior facilidade para revisão e alterações & 29 & 100,0 & 0 & 0,0 & 0 & 0,0 & 0 & 0,0 \\
\hline Alta precisão & 25 & 86,2 & 2 & 6,9 & 2 & 6,9 & 0 & 0,0 \\
\hline Maior possibilidade de avaliação de desenhos alternativos & 23 & 79,3 & 0 & 0,0 & 4 & 13,8 & 2 & 6,9 \\
\hline Uso de partes comuns em múltiplos produtos & 27 & 93,1 & 0 & 0,0 & 1 & 3,4 & 1 & 3,4 \\
\hline Maior garantia de qualidade dos produtos & 24 & 82,8 & 3 & 10,3 & 2 & 6,9 & 0 & 0,0 \\
\hline Economia direta de custo no projeto & 27 & 93,1 & 2 & 6,9 & 0 & 0,0 & 0 & 0,0 \\
\hline Melhoria da qualidade do projeto, em termos de resultado & 29 & 100,0 & 0 & 0,0 & 0 & 0,0 & 0 & 0,0 \\
\hline $\begin{array}{l}\text { Desenvolvimento profissional do pessoal de } \\
\text { projeto envolvido em CAD }\end{array}$ & 23 & 79,3 & 3 & 10,3 & 3 & 10,3 & 0 & 0,0 \\
\hline Aumento da produtividade do pessoal envolvido em projeto & 28 & 96,6 & 0 & 0,0 & 1 & 3,4 & 0 & 0,0 \\
\hline Melhoria nas comunicações entre pessoal de projeto & 19 & 65,5 & 6 & 20,7 & 4 & 13,8 & 0 & 0,0 \\
\hline Aumentos salariais para usuários de sistemas CAD & 6 & 20,7 & 16 & 55,2 & 7 & 24,1 & 0 & 0,0 \\
\hline Aquisição de novos conhecimentos para o pessoal de projeto & 21 & 72,4 & 6 & 20,7 & 2 & 6,9 & 0 & 0,0 \\
\hline Melhor qualidade da documentação & 27 & 93,1 & 2 & 6,9 & 0 & 0,0 & 0 & 0,0 \\
\hline Diminuição do número de cópias de desenho em papel & 18 & 62,1 & 11 & 37,9 & 0 & 0,0 & 0 & 0,0 \\
\hline Centralização das informações de projeto & 21 & 72,4 & 6 & 20,7 & 2 & 6,9 & 0 & 0,0 \\
\hline Melhoria no fluxo de informações através da empresa & 20 & 69,0 & 5 & 17,2 & 4 & 13,8 & 0 & 0,0 \\
\hline Uso de bibliotecas-padrão para rápida repetição de cópias & 27 & 93,1 & 0 & 0,0 & 2 & 6,9 & 0 & 0,0 \\
\hline
\end{tabular}

tais melhorias, ainda há uma parcela relevante de empresas onde não se vislumbram tais aspectos da utilização de sistemas CAD. E a centralização das informações de projeto com sistemas CAD na empresa foi verificada em $73 \%$ das empresas; contudo, em 20\% delas não houve centralização das informações; e em $7 \%$ não se aplica tal vantagem relacionada aos sistemas CAD. Assim, da mesma forma que as melhorias, existe uma parcela significativa de empresas que não contemplam a centralização das informações de projeto com sistemas CAD. Dessa maneira, parece ainda haver uma deficiência no gerenciamento de informações e na comunicação relacionada à utilização de sistemas CAD.

Caulliraux e Costa (1995) e Romeiro Filho (1997) mencionam a necessidade da padronização de métodos e procedimentos do projeto para que o uso de sistemas CAD traga benefícios para a organização. Aqui, observou-se que, na maioria das empresas (83\%), há a padronização de métodos e procedimentos do projeto com sistemas CAD. Portanto, esse dado leva a crer que as empresas já notaram a necessidade de padronização de métodos e procedimentos e a colocaram em prática como forma de melhor utilizar os sistemas CAD em projeto.
Autores Caulliraux e Costa (1995) mencionam a necessidade do desenvolvimento profissional do pessoal de projeto envolvido em sistemas CAD na empresa e da aquisição de novos conhecimentos por esse pessoal de projeto, já que os recursos humanos são essenciais para uso da tecnologia na área de projeto das empresas, representada pelos sistemas CAD. Dessa forma, verificou-se o desenvolvimento profissional do pessoal de projeto envolvido em sistemas CAD em 77\% das empresas. Já a aquisição de novos conhecimentos para o pessoal de projeto com sistemas CAD na empresa ocorreu em 70\% das empresas.

Assim, com dados aproximadamente similares, pode-se dizer que o desenvolvimento profissional e a aquisição de novos conhecimentos parecem acontecer na maior parte das empresas mineiras do setor metal-mecânico, contudo, há indícios de que parcela significativa dessas empresas não disponha de eficientes mecanismos ou programas de treinamento e de desenvolvimento profissional para a mão de obra envolvida em atividades de projeto. 0 que é reforçado pelos dados obtidos sobre os pesquisados a respeito da formação em sistemas CAD nas empresas. Outro aspecto pesquisado que reforça essa ineficiência de mecanismos ou 
programas de desenvolvimento profissional em parcela significativa das empresas foi a ocorrência de aumentos salariais para usuários de sistemas $C A D$ na empresa; porque observou-se aumentos salariais em apenas 20\% das empresas; em 53\% delas, não houve aumentos para usuários de sistemas CAD; e em $27 \%$ isso não se aplica. Portanto, na maior parte das empresas ou não há ou se aplica aumentos salariais para usuários de sistemas CAD.

\subsection{Desvantagens na utilização dos sistemas $C A D$}

A grande maioria (87\%) dos pesquisados considerou que ou não há ou são poucas as desvantagens da adoção de sistemas CAD na área de projeto da empresa, o que também reforça o papel fundamental assumido pela utilização de sistemas CAD para projeto de produtos dentro das organizações do setor metal-mecânico de Minas Gerais. Os problemas de ordem técnica são um dos aspectos importantes a serem tratados quando se estuda a utilização de sistemas CAD e acabam se tornando desvantagens desses sistemas. Podem-se citar alguns desses problemas técnicos com o endosso de Romeiro Filho (1997): tempo perdido (down-time) devido a falhas no equipamento com sistemas $C A D$ na empresa; espera na fila devido a outras requisições de uso das estações gráficas com sistemas CAD; lentidão do equipamento para trabalho gráfico; manutenção deficiente ou inexistente; falta de equipamentos importantes para o funcionamento do sistema $\mathrm{CAD}$; incompatibilidade entre equipamentos e/ou softwares CAD; ausência de softwares adequados ao trabalho proposto e ausência de banco de dados e bibliotecas de símbolos. Evidentemente, esses problemas que se configuram como desvantagens do uso de sistemas também foram examinados pelo survey, conforme demonstra a Tabela 2 .

Com relação à ausência de softwares adequados ao trabalho proposto, na grande maioria das empresas não se verificou (83\%) ou não se aplica (7\%) a ausência de programas para o trabalho proposto em projeto. 0 que parece indicar que as empresas pesquisadas estão superando um dos problemas apontados por Figueiredo, Lacerda e Romeiro (2003): a adoção equivocada de sistemas CAD para um determinado conjunto de atividades de projeto. Esses autores também apontam para outro problema: o alto custo do equipamento para sistemas CAD e treinamento adicional, observados em 67\% das empresas, o que sugere que a maioria das empresas ainda considera elevado o gasto para investir em sistemas CAD.
Diversos autores, como Romeiro Filho (1997) e Dankwort et al. (2004), alertam para outro problema relacionado à adoção de sistemas CAD: a dificuldade de aprendizado para utilização do equipamento hardware e para utilização do software gráfico. Entretanto, a dificuldade de aprendizado para utilização do equipamento hardware apresentou comportamento diferente do comportamento da dificuldade de aprendizado para utilização do software gráfico, como demonstram os dados coletados e mostrados no item anterior. Esses dados indicam que, na maioria das empresas (73\%), não houve dificuldade no aprendizado para utilização do equipamento hardware; enquanto em 57\% delas não houve dificuldade de aprendizado para utilização do software gráfico. Contudo, em 17\% das empresas observou-se dificuldade no aprendizado para utilização do equipamento hardware; enquanto em 33\% delas observou-se dificuldade de aprendizado para utilização do software gráfico. Assim, parece que os usuários dos sistemas CAD dentro das empresas superaram a dificuldade de aprendizado para utilização do equipamento hardware, mas ainda apresentam dificuldade de aprendizado para utilização do software gráfico.

No que se refere às frequentes modificações no software gráfico, que dificultam o aprendizado, constatou-se que na maioria das empresas não houve modificações (77\%). Já no tocante ao sistema de suporte e orientação aos usuários deficiente ou inexistente e à falta de informação sobre o sistema, suas aplicações e formas de utilização, os dados coletados se mostraram inconcludentes. Observou-se que em 37\% das empresas o sistema de suporte e orientação aos usuários é deficiente ou inexistente, e em $40 \%$ das empresas houve falta de informação sobre o sistema, suas aplicações e formas de utilização, e ainda em 53\% delas o sistema de suporte e orientação aos usuários não é deficiente ou inexistente e em 50\% não houve falta de informação sobre o sistema, suas aplicações e formas de utilização; em ambos os casos em 10\% das empresas não se aplicam tais desvantagens. Soma-se a isso que os dados coletados sobre a inexistência de programas formais e estruturados de treinamento também se mostram inconcludentes, já que em 37\% das empresas constatou-se essa inexistência; em 53\% delas não se constatou a inexistência, e ainda em 10\% não se aplica tal desvantagem relacionada aos sistemas CAD. Então, a análise desses dados sobre modificações no software gráfico, sobre o sistema de suporte e orientação aos usuários e informação sobre o sistema, suas aplicações e formas de utilização e sobre programas 
Tabela 2. Desvantagens na utilização do CAD nas empresas pesquisadas.

\begin{tabular}{|c|c|c|c|c|c|c|c|c|}
\hline \multirow[t]{2}{*}{ Principais desvantagens dos sistemas CAD na empresa } & \multicolumn{2}{|c|}{ Sim } & \multicolumn{2}{|c|}{ Não } & \multicolumn{2}{|c|}{$\begin{array}{l}\text { Não se } \\
\text { aplica }\end{array}$} & \multicolumn{2}{|c|}{ Não sabe } \\
\hline & $\mathrm{N}^{\circ}$ & $\%$ & $\mathrm{~N}^{\mathrm{o}}$ & $\%$ & $\mathrm{~N}^{\circ}$ & $\%$ & $\mathrm{~N}^{\circ}$ & $\%$ \\
\hline Tempo perdido (down-time) devido a falhas no equipamento & 6 & 20,7 & 19 & 65,5 & r & 13,8 & 0 & 0,0 \\
\hline Espera na fila devido a outras requisições de uso das estações gráficas & 2 & 6,9 & 20 & 69,0 & 6 & 20,7 & 1 & 3,4 \\
\hline Alto custo do equipamento e treinamento adicional & 19 & 65,5 & 7 & 24,1 & 2 & 6,9 & 1 & 3,4 \\
\hline Equipamento lento para trabalho gráfico (demora de resposta pelo computador) & 7 & 24,1 & 17 & 58,6 & 5 & 17,2 & 0 & 0,0 \\
\hline Difícil aprendizado para utilização do equipamento hardware & 5 & 17,2 & 21 & 72,4 & 3 & 10,3 & 0 & 0,0 \\
\hline Manutenção deficiente ou inexistente, gerando problemas técnicos frequentes & 3 & 10,3 & 21 & 72,4 & 5 & 17,2 & 0 & 0,0 \\
\hline Falta de equipamentos importantes para o funcionamento do sistema CAD & 5 & 17,2 & 20 & 69,0 & 4 & 13,8 & 0 & 0,0 \\
\hline Incompatibilidade entre equipamento e/ou software & 5 & 17,2 & 21 & 72,4 & 3 & 10,3 & 0 & 0,0 \\
\hline Difícil aprendizado para utilização do software gráfico & 9 & 31,0 & 17 & 58,6 & 3 & 10,3 & 0 & 0,0 \\
\hline Frequentes modificações no software gráfico dificultam o aprendizado & 4 & 13,8 & 22 & 75,9 & 3 & 10,3 & 0 & 0,0 \\
\hline Sistema de suporte e orientação aos usuários deficiente ou inexistente & 11 & 37,9 & 15 & 51,7 & 3 & 10,3 & 0 & 0,0 \\
\hline Falta de informações sobre o sistema, suas aplicações e formas de utilização & 12 & & 14 & & 3 & 10,3 & 0 & 0,0 \\
\hline Ausência de softwares adequados ao trabalho proposto & 3 & 10,3 & 24 & 82,8 & 2 & 6,9 & 0 & 0,0 \\
\hline Ausência de bancos de dados e bibliotecas de símbolos & 10 & 34,5 & 17 & 58,6 & 2 & 6,9 & 0 & 0,0 \\
\hline Fadiga óptica dos usuários & 13 & 44,8 & 11 & 37,9 & 2 & 6,9 & 3 & 10,3 \\
\hline Problemas de postura (dores nas costas, pescoço etc.) & 19 & 65,5 & 6 & 20,7 & 1 & 3,4 & 3 & 10,3 \\
\hline Aumento nos níveis de tensão durante o trabalho & 8 & 27,6 & 17 & 58,6 & 2 & 6,9 & 2 & 6,9 \\
\hline Trabalho constante em ambientes refrigerados, demasiadamente frios & 3 & 10,3 & 22 & 75,9 & 3 & 10,3 & 1 & 3,4 \\
\hline lluminação muitas vezes deficiente para trabalho em estações gráficas & 6 & 20,7 & 20 & 69,0 & 2 & 6,9 & 1 & 3,4 \\
\hline Desemprego para o pessoal de projeto a partir da implantação do CAD & 4 & 13,8 & 21 & 72,4 & 3 & 10,3 & 1 & 3,4 \\
\hline Perda de conhecimentos adquiridos nas atividades em prancheta & 8 & 27,6 & 19 & 65,5 & 2 & 6,9 & 0 & 0,0 \\
\hline Inexistência de programas formais e estruturados de treinamento & 11 & 37,9 & 15 & 51,7 & 3 & 10,3 & 0 & 0,0 \\
\hline Resistências ao CAD por parte do pessoal técnico & 3 & 10,3 & 24 & 82,8 & 2 & 6,9 & 0 & 0,0 \\
\hline Resistências ao CAD por parte do corpo gerencial e administrativo & 5 & 17,2 & 22 & 75,9 & 2 & 6,9 & 0 & 0,0 \\
\hline Problemas de cultura da empresa, pouco preparada p/ inovações como o CAD & 6 & 20,7 & 19 & 65,5 & 4 & 13,8 & 0 & 0,0 \\
\hline Quedas nos níveis salariais do pessoal de projeto envolvidos com CAD & 3 & 10,3 & 23 & 79,3 & 2 & 6,9 & 1 & 3,4 \\
\hline Transformação do profissional de projeto em mero operador do sistema CAD & 6 & 20,7 & 20 & 69,0 & 2 & 6,9 & 1 & 3,4 \\
\hline
\end{tabular}

formais e estruturados de treinamento reforça que um dos mais importantes obstáculos à utilização de sistemas $C A D$ reside na ineficiência de mecanismos ou programas de treinamento e de desenvolvimento profissional para a mão de obra envolvida em atividades de projeto.

\section{Conclusões}

A pesquisa demonstrou que as empresas dominam uma aplicação básica dos sistemas CAD, a modelagem geométrica, conforme aponta a literatura, e fazem uso dela para a realização de desenhos mecânicos e para a sua complementação com desenhos auxiliares. Demonstrou também que as aplicações mais complexas e amplas são empreendidas apenas por parcela das empresas. Por outro lado, uma parcela das empresas também usa os sistemas CAD para a elaboração de textos, gráficos e/ou planilhas, em um aparente desvio de aplicação.
Algumas empresas possuem implementações de sistemas CAD bastante desenvolvidas, verificando-se nelas aspectos como maior velocidade de projetos, maior integração entre projetistas e equipes, redução no trabalho de desenho, menor tempo e maior facilidade para revisão e alterações, maior produtividade, precisão, uso de partes comuns e bibliotecas-padrão, economia direta de custo e qualidade. Esses aspectos são coerentes com as vantagens apresentadas pela literatura referenciada com relação à utilização de sistemas informatizados de apoio ao projeto.

Contudo, quando se abordam outros aspectos, como melhoria das comunicações entre o pessoal de projeto, melhoria no fluxo de informações através da empresa com sistemas CAD e centralização das informações de projeto com sistemas $C A D$, ainda há uma parcela relevante de empresas onde não se vislumbram tais aspectos. E, ainda, mecanismos ou programas de treinamento e de desenvolvimento profissional para a mão de obra envolvida com CAD se demonstram pouco eficientes. Esses aspectos 
podem ser avaliados de forma conjunta, o que leva à necessidade de revisão dos processos de formação de usuários diretos (operadores) ou indiretos (gerentes, por exemplo). Nesse caso, a formação deverá enfocar não somente aspectos "operacionais" dos sistemas, mas também os impactos dessa tecnologia na estrutura e organização da empresa como um todo; incluindo possibilidades de integração com outros grupos além dos envolvidos com projeto, tentando alcançar um ambiente propício à Engenharia Simultânea e ao PLM.

Entretanto, a pesquisa demonstra que ainda existe um longo caminho pela frente. Embora grande parte das empresas utilize sistemas CAD a um tempo considerado longo, a tecnologia ainda não se encontra desenvolvida na maioria das empresas no que se refere a aplicações consideradas mais complexas, envolvendo conceitos como PLM. Nesse caso, aparentemente a questão se torna mais voltada à gestão do processo de projeto do que ao uso da ferramenta CAD. Nesse sentido, futuros estudos deverão envolver questões ligadas à gestão do conhecimento a partir da área de projetos e como essa gestão pode contribuir na melhoria dos resultados da empresa no que diz respeito ao projeto de novos produtos.

Portanto, o processo de aumento de competitividade afeta a indústria como um todo e envolve diferentes iniciativas relacionadas ao design e projeto de produtos, à tecnologia e aos recursos humanos que estão em curso nas empresas brasileiras. E nesse cenário, os sistemas CAD assumem papel de grande importância, devido ao seu potencial como ferramenta para agilizar e unificar o processo projetual, bem como na integração do projeto com outras áreas da empresa. Dessa forma, a utilização desses sistemas apoiando o design e o projeto de produto foi uma das formas encontradas para que fossem alcançados padrões técnicos e de produtividade compatíveis com condições de competitividade em que se inserem empresas do Brasil.

\section{Referências}

AMANN, K. PDM to PLM: envolving to the future. 2004 Disponivel em: <http://www.cimdata.com/>. Acesso em: 3 dez. 2009.

ANUMBA, C. J. Functional integration in CAD systems. Advances in Engineering, v. 25, p. 103-109, 1996. doi:10.1016/0965-9978(95)00102-6

BARBETTA, P. A. Estatística aplicada às ciências sociais. 5. ed. Florianópolis: Ed. UFSC, 2002.

CAULLIRAUX, H. M.; COSTA, L. S. S. (Org.). Manufatura integrada por computador. sistemas integrados de produção: estratégia, organização, tecnologia e recursos humanos. Rio de Janeiro: Ed. Campus, 1995. 450 p.

CIMDATA. All about PLM: Product lifecycle Management (PLM) definition. Disponível em: <http://www.cimdata. com $/ \mathrm{plm} . \mathrm{html}>$. Acesso em: 5 dez. 2009.

CLARK, K. B.; FUJIMOTO, T. Product development performance: strategy, organization, and management in the world auto industry. Boston: Harvard Business School, 1991.

CLARK, K. B.; WHEELWRIGHT, S. C. Managing new product and process development: text and cases. New York: Harvard Business School, 1993.

CONFEDERAÇÃO NACIONAL DA INDÚSTRIA - $\mathrm{CNI}$ Indicadores de competitividade na indústria brasileira. 2. ed. Brasília: CNl, 2005. 126 p.

DANKWORT, C. W. et al. Engineers' CAx education-it's not only CAD. Computer-Aided Design, v. 36, p. 1439-1450, 2004. doi:10.1016/j.cad.2004.02.011

DAVIES, B. J. CIM Software and interfaces. Computer in Industry, v. 33, p. 91-99, 1997. doi:10.1016/S01663615(97)00014-6

FEDERAÇÃO DAS INDÚSTRIAS DO ESTADO DE MINAS GERAIS - FIEMG. Perfil da economia mineira. Belo Horizonte: Gerência de Economia, Federação das Indústrias do Estado de Minas Gerais, 2006a. Disponível em: <http://www.fiemg.com.br/content/diretoria/ default.asp?cod_pai $=86 \&$ cod_diretoria $=214 \&$ nom diretoria=lnformações Econômicas\#>. Acesso em: 1 jun. 2007.

FEDERACÃO DAS INDÚSTRIAS DO ESTADO DE MINAS GERAIS - FIEMG. FIEMG index: relatório da pesquisa de indicadores industriais. Belo Horizonte: FIEMG, 2006b. Disponível em: <http://www.fiemg.com.br/content/ diretoria/default.asp?cod_pai $=86 \&$ cod_diretoria $=214$ $\&$ nom_diretoria $=$ InformaçõesEconômicas\# $>$. Acesso em: 19 dez. 2009.

FIGUEIREDO, A. C.; LACERDA, D. C.; ROMEIRO FILHO, E. A implantação de sistemas informatizados de apoio ao projeto e seus principais problemas: um estudo a partir do ponto de vista dos fornecedores. In: CONGRESSO BRASILEIRO DE ENGENHARIA DE FABRICAÇÃO COBEF, 2. Anais... Uberlândia: Associação Brasileira de Ciências Mecânicas, 2003.

FLEURY, A. Gerenciamento do desenvolvimento de produtos na economia globalizada. In: CONGRESSO BRASILEIRO DE GESTÃO DE DESENVOLVIMENTO DE PRODUTO, 1. 1999, Belo Horizonte. Anais..

FORZA, C. Survey research in operations management: a process-based perspective. International Journal of Operations and Production Management, vol. 22, n. 2, p. 152-194, 2002.

GRIFFIN, A. PDMA research on new product development practices: updating trends and benchmarking best practices. Journal of Product Innovation Management, v. $18,1997$.

GRIFFIN, A.; PAGE, A. L. PDMA success measurement project: recommended measures for product development sucess and failure. Journal of Product Innovation Management, v. 13, p. 478-496, 1996. doi:10.1016/ S0737-6782(96)00052-5

JON PEDDIE RESEARCH - JPR. CAD Report. 2010. Disponível em: $\quad<$ http://www.jonpeddie.com/publications/cad report>. Acesso em: 21 maio 2010. 
KRISHNAN, V.; ULRICH, K. T. Product development decisions: a review of the literature. Management Science, v. 47, n. $1,2001$.

LUONG, L. H. S. A decision support system for the selection of computer-integrated manufacturing technologies. Robotics an Computer-Integrated Manufacturing, n. 14, p. 45-53, 1998. doi:10.1016/S0736-5845(97)00026-4

MALHOTRA, M. K.; HEINE, M. L.; GROVER, V. An evaluation of the relationship between management practices and computer aided design technology. Journal of Operations Management, v. 19, p. 307-333, 2001. doi:10.1016 S0272-6963(00)00063-2

McELENEY, J. J. PDMvsPLM Byline. 2006. Disponível em: <http://globalsite.solidworks.com/ brazil/files/ PDMvsPLM.pdf>. Acesso em: 15 dez. 2009.

NAGALINGAM, S. V.; LIN G. C. I. CIM - still the solution for manufacturing industry. Robotics and ComputerIntegrated Manufacturing, v. 24, p. 332-344, 2008. doi:10.1016/j.rcim.2007.01.002

NEVES, M.; CAULlIRAUX, H. M.; SOUZA, A. Sistemas integrados de produção no brasil: os esforços na área de recursos humanos. Revista Produto e Produção, n. 2, 1998.

PAHL, G. et al. Projeto na engenharia: fundamentos do desenvolvimento eficaz de produtos, métodos e aplicações. São Paulo: Edgard Blücher, 2005. 412 p.

PAHL, G.; BEITZ, W. Engineering design: a systematic approach. London: Springer-Verlag, 1996.

PINSONNEAULT, A.; KRAEMER, K. L. Survey research methodology in management information systems: an assessment. Journal of Management Information Systems, v. 10, n. 2, p.75-105, 1993.

QIU, Z. M. et al. Role-based 3D visualization for asynchronous PLM collaboration. Computers in Industry, v. 58, p. 747755, 2007. doi:10.1016/j.compind.2007.02.006

REHG, J. A.; KRAEBBER, H. W. Computer-integrated manufacturing. New Jersey: Prentice Hall, 2005. 574 p.

REMBOLD, U.; NNAJl, B. 0.; STORR, A. Computer integrated manufacturing and engineering. UK: Addison-Wesley, 1993. $640 \mathrm{p}$.
ROMEIRO FILHO, E. CAD na indústria: implantação e gerenciamento. Rio de Janeiro: Ed. UFRJ, 1997. 180 p.

ROOZENBURG, N. F. M.; EEKELS, J. Product design: fundamentals and methods. Nova lorque: John Wiley \& Sons, 1995. 408 p.

ROSENTHAL, S. R. Progress toward the 'factory of the future'. Journal of Operations Management, v. 4, n. 3, 1984.

SCHEER, A. W. CIM: evoluindo para a fábrica do futuro. Rio de Janeiro: Qualitymark, 1993. 228 p.

SHEAFFER, R. L.; MENDENHALL, W.; OTTO, L. Elementary survey sampling. $4^{\text {th }}$ ed. Belmont: Duxbury, 1990. $389 \mathrm{p}$.

SOLIMAN, F.; CLEGG, S. Critical success factors for integration of CAD/CAM systems with ERP systems. International Journal of Operations \& Production Management, v. 21, n. 5/6, p. 609-629, 2001. doi:10.1108/01443570110390363

STAMATI, V.; FUDOS, 1. A parametric feature-based CAD system for reproducing traditional pierced jewellery. Computer-Aided Design, v. 37, p. 431-449, 2005. doi:10.1016/j.cad.2004.07.004

TAFFS, D. Case study - client and project. Automation in Construction, n. 6, p. 59-64, 1997. doi:10.1016/S09265805(96)00182-3

ULRICH, K. T.; EPPINGER, S. D. Product design and development. International Edition. Boston: McGrawHill, 2000. 358 p.

URBAN, G. L.; HAUSER, J. R. Design and marketing of new products. New Jersey: Prentice-Hall, 1993. 701 p.

\section{Agradecimentos}

Os autores agradecem ao CNPq, CAPES, FAPEMIG e FINEP, que apoiaram financeiramente diferentes etapas deste trabalho, bem como as empresas e seus empregados que gentilmente colaboraram para a pesquisa. Por fim, agradecem os referees que prestaram valiosa colaboração com a revisão do artigo.

\title{
The contribution of CAD practices: a survey on the metal-mechanic industry in the State of Minas Gerais
}

\begin{abstract}
This article has the objective to expose the accomplishments of a descriptive survey for the evaluation of the use of CAD (Computer Aided Design) systems in product projects in organizations of the metal-mechanic sector in the State of Minas Gerais, Brazil. As a result of the survey, differences were observed between the CAD applications described in the literature on CAD systems in Product Design and the project practices aided by computer in the researched organizations as a result of specific characteristics of the area and the studied sector. On the other hand, the results show that problems presented in the literature review are the same ones observed in the industries, what can indicate an improvement possibility to CAD applications, through an adequate approach.
\end{abstract}

Keywords

CAD. Product project. Survey. Metal-mechanic section. 